\title{
The Cosmic Large-Scale Structure in X-rays (CLASSIX) Cluster Survey
}

\section{The Perseus-Pisces supercluster and the Southern Great Wall as traced by $\mathrm{X}$-ray luminous galaxy clusters ${ }^{\star}$}

\author{
Hans Böhringer ${ }^{1,2}$, Gayoung Chon ${ }^{1}$, and Joachim Trümper ${ }^{2}$ \\ ${ }^{1}$ Universitäts-Sternwarte München, Fakultät für Physik, Ludwig-Maximilians-Universität München, Scheinerstr. 1, 81679 München, \\ Germany \\ 2 Max-Planck-Institut für Extraterrestrische Physik, 85748 Garching, Germany \\ e-mail: hxb@mpe.mpg.de
}

Received 23 March 2021 / Accepted 6 May 2021

\begin{abstract}
The Perseus-Pisces supercluster is known as one of the largest structures in the nearby Universe that has been charted by the galaxy and galaxy cluster distributions. For the latter mostly clusters from the Abell catalogue have been used. Here we take a new approach to a quantitative characterisation of the Perseus-Pisces supercluster using a statistically complete sample of X-ray luminous galaxy groups and clusters from our CLASSIX galaxy cluster redshift survey. We used a friends-of-friends technique to construct the supercluster membership. We also studied the structure of the Southern Great Wall, which merges with the Perseus-Pisces supercluster with a slightly increased friends-of-friends linking length. In this work we discuss the geometric structure of the superclusters, compare the $\mathrm{X}$-ray luminosity distribution of the members with that of the surroundings, and provide an estimate of the supercluster mass. These results establish Perseus-Pisces as the largest superstructure in the Universe at redshifts $z \leq 0.03$. With the new data this supercluster extends through the zone of avoidance, which has also been indicated by some studies of the galaxy distribution by means of HI observations. We investigated whether the shapes of the member groups and clusters in X-rays are aligned with the major axis of the supercluster. We find no evidence for a pronounced alignment, except for the ellipticities of Perseus and AWM7, which are aligned with the separation vector of the two systems and weakly with the supercluster.
\end{abstract}

Key words. galaxies: clusters: general - cosmology: observations - large-scale structure of Universe - X-rays: galaxies: clusters

\section{Introduction}

The large-scale structure of the Universe is often described as the cosmic web traced by galaxies and galaxy clusters. In the hierarchy of structures superclusters (SCs) are the next largest units above galaxy clusters (e.g. Bahcall 1988; Einasto et al. 2007). The term superclusters is not very well defined, and is applied to a variety of structures with a large range of sizes and overdensities. We therefore introduced a clearer definition of superclusters at higher overdensities, comprising those supercluster mass distributions that will collapse in the future in a $\Lambda$ cold dark matter $(\Lambda \mathrm{CDM})$ Universe, calling them superstes-clusters (Chon et al. $2015)^{1}$. Structures larger than this will be transient phenomena in the long run and we refer to them here as superclusters. They are still in the regime of non-linear structures, as reflected in their flattened or filamentary shapes and overdensities well exceeding unity. On scales larger than $\sim 300 \mathrm{Mpc}$, the density fluctuations approach a Gaussian distribution and the statistics can be fairly well described by the matter distribution power spectrum. In this

\footnotetext{
* Based on observations at the European Southern Observatory La Silla, Chile, and the German-Spanish Observatory at Calar Alto.

1 Superstes is Latin for survivor, and describes the fate of these structures that will survive a dispersion in a Universe with accelerated expansion.
}

paper and the other publications in this series we study the superclusters in the nearby Universe using galaxy clusters as probes.

In this paper we characterise the Perseus-Pisces SC and the neighbouring Southern Great Wall (SGW). The PerseusPisces SC was found to be one of the largest SCs in the early exploration of the cosmic web (e.g. Joeveer \& Einasto 1978; Gregory et al. 1981; Chincarini et al. 1983; Batuski \& Burns 1985). Joeveer \& Einasto (1978) found the Perseus-Pisces SC to be the most prominent structure in the Universe out to a recession velocity of $15000 \mathrm{~km} \mathrm{~s}^{-1}\left(\sim 214 h_{70}^{-1} \mathrm{Mpc}\right)$ among other chains of clusters in the constellations Coma, Hercules, and Fornax. They describe the Perseus-Pisces SC to cover about $45^{\circ}$ of the sky comprising the clusters Abell 426 (Perseus), A 262, A 347 and the galaxy groups associated with the giant galaxies NGC 315, NGC 383, NGC 507, and NGC 1129. Based on a total luminosity of about $10^{13} h_{50}^{-2} L_{\odot}$ they attribute a mass of about $2 \times 10^{16} h_{50}^{-1} M_{\odot}$ to this SC and find that it has an overdensity compared to the cosmic mean of about a factor of 3 . They remark that all member clusters are elongated along the main ridge of the chain.

Gregory et al. (1981) described the Perseus-Pisces SC as a concentration of clusters on an area of about $40 \times 12^{\circ}$ on the sky, occupying a volume of about $2 \times 10^{5} h^{-3} \mathrm{Mpc}^{3}$ at a mean redshift of $z=0.0178$. They associate similar members with the SC, as did Joeveer \& Einasto. Batuski \& Burns (1985) isolated a larger 
structure in their study out to $z=0.08$, named the Perseus-Pegasus filament, with an extent of $300 \mathrm{Mpc}$. This structure is much larger than the Perseus-Pisces SC considered here; it extends into the constellation of Pegasus and Aquarius and reaches higher redshifts than the structures described above. More recent works (e.g. Hauschildt 1987; Chamaraux et al. 1990; Ramatsoku et al. 2016; Kraan-Korteweg et al. 2018) based on radio observations of $\mathrm{HI}$ in galaxies discuss various extensions of the Perseus-Pisces SC, also into the zone of avoidance (ZoA), which is discussed in Sect. 5.

In this study we used X-ray luminous galaxy clusters for the large-scale structure mapping. Since clusters form from the largest peaks in the initially random Gaussian density fluctuation field, their density distribution can be statistically closely related to the matter density distribution (e.g. Bardeen et al. 1986). Using X-ray luminosity to characterise the clusters has two advantages. The X-ray emission ensures that the systems are tight three-dimensional mass concentrations, and the X-ray luminosity provides a good estimate of the object's mass.

Cosmic structure formation theory has shown that the ratio of the cluster density fluctuation amplitude is biased with respect to the matter density fluctuations in the sense that the cluster density fluctuations follow the matter density fluctuations with a larger amplitude. This bias is practically scale-independent (e.g. Kaiser 1986; Mo \& White 1996; Sheth \& Tormen 1999; Tinker et al. 2010). The bias of the cluster density fluctuation amplitude is an enhancement, which makes clusters sensitive tracers of the matter distribution.

For our study we used the large, highly complete sample of $\mathrm{X}$-ray luminous galaxy clusters from the Cosmic Large-Scale Structure in X-rays (CLASSIX) galaxy cluster survey. The survey is flux-limited, which provides an X-ray luminosity-limited (and closely mass-limited) cluster sample in each redshift shell. We have already applied CLASSIX to successful studies of the cosmic large-scale structure, as explained in Sect. 2, and the present study can build on this experience.

The paper has the following structure. In Sect. 2 we describe the CLASSIX galaxy cluster survey. Section 3 deals with methodological aspects. The results of our analysis is presented in Sect. 4. Section 5 provides a discussion. A summary and conclusions are given in Sect. 6. In the appendix we provide combined X-ray and optical images of the members of the PerseusPisces SC and the SGW, investigate the alignment of cluster shapes with the SC main axis in more detail, and discuss the morphology of one of the most compact groups in our sample, NGC 410. For physical properties that depend on distance we use the following cosmological parameters: $H_{0}=70 \mathrm{~km} \mathrm{~s}^{-1} \mathrm{Mpc}^{-1}$, $\Omega_{\mathrm{m}}=0.3$, and a spatially flat metric. For the cosmographical analysis we use Supergalactic coordinates, defined by the location of the Supergalactic north pole at $l_{\mathrm{II}}=47.3700^{\circ}$ and $b_{\mathrm{II}}=6.3200^{\circ}$, as established by de Vaucouleurs et al. (1991) in the Third Catalogue of Bright Galaxies (see also Lahav et al. 2000). For the $X$-ray luminosities quoted in the following we use the ROSAT band, $0.1-2.4 \mathrm{keV}$.

\section{The CLASSIX galaxy cluster survey}

The CLASSIX galaxy cluster catalogue provides an ideal database for this study since the cluster density is high enough to provide a meaningful dense sampling of the large-scale matter distribution and the selection function is well understood, allowing an unbiased mapping of this distribution. CLASSIX is the combination of our surveys in the southern sky, REFLEX II (Böhringer et al. 2013), and the northern hemisphere, NORAS II (Böhringer et al. 2017). The total coverage is 8.26 ster of the sky at galactic latitudes $\left|b_{\mathrm{II}}\right| \geq 20^{\circ} 1$. An extension of CLASSIX also includes lower galactic latitudes, part of the ZoA, in a region restricted to interstellar hydrogen column density $n_{\mathrm{H}} \leq 2.5 \times 10^{21} \mathrm{~cm}^{-2}$. At higher column density X-rays are strongly absorbed in the ROSAT energy band, and the sky usually has a high stellar density, making the detection of clusters in the optical extremely difficult. The values for the interstellar hydrogen column density are taken from the $21 \mathrm{~cm}$ survey of Dickey \& Lockman $(1990)^{2}$. This area adds another 2.56 ster and increases the sky coverage to $86.2 \%$ of the sky. The completeness of the cluster detection in this area is not as high as for REFLEX and NORAS, and follow-up to obtain redshifts for this part of the survey is still incomplete. The statistical properties of the cluster distribution in the ZoA are therefore somewhat qualitative. In addition, three known X-ray luminous clusters in the region with $n_{\mathrm{H}}>2.5 \times 10^{21} \mathrm{~cm}^{-2}$, which fulfil the X-ray selection parameters, were added to the cluster sample.

The CLASSIX galaxy cluster survey and its extension is based on the X-ray detection of galaxy clusters in the ROSAT All-Sky Survey (RASS, Trümper 1993; Voges et al. 1999). The construction of the cluster survey and the survey selection function as well as tests of the completeness are described in Böhringer et al. (2013, 2017). In summary, the nominal unabsorbed flux limit for the galaxy cluster detection in the RASS is $1.8 \times 10^{-12} \mathrm{erg} \mathrm{s}^{-1} \mathrm{~cm}^{-2}$ in the $0.1-2.4 \mathrm{keV}$ energy band, and we require a minimum count of detected source photons of 20 . Under these conditions the nominal flux limit quoted above is reached in about $80 \%$ of the survey. In regions with lower exposure and higher interstellar absorption the flux limit is accordingly higher (see Fig. 11 in Böhringer et al. 2013 and Fig. 5 in Böhringer et al. 2017), and this effect is well modelled and taken into account in the survey selection function.

We already applied the REFLEX I (Böhringer et al. 2004) and REFLEX II surveys to study the cosmic large-scale matter distribution, for example through the correlation function (Collins et al. 2000), the power spectrum (Schuecker et al. 2001, 2002, 2003a,b; Balaguera-Antolinez et al. 2011, 2012), and Minkowski functionals (Kerscher et al. 2001). We compiled a catalogue of superstes-clusters and studied their properties (Chon \& Böhringer 2013; Chon et al. 2014). We find from the cluster distribution that the local Universe has a lower matter density inside a radius of $\sim 100-170 \mathrm{Mpc}$ than the cosmic mean on larger scales (Böhringer et al. 2015, 2020), and we show that on a scale of $\sim 100 \mathrm{Mpc}$ the matter distribution is strongly segregated onto the Supergalactic plane (Böhringer et al. 2021).

The two most essential physical parameters of the clusters are their X-ray luminosity and mass. X-ray luminosities in the $0.1-2.4 \mathrm{keV}$ energy band were derived within a cluster radius of $r_{500}{ }^{3}$. To estimate the cluster mass and temperature from the observed X-ray luminosity, we use the scaling relations described in Pratt et al. (2009). They were determined from a representative cluster sub-sample of our survey called REXCESS (Böhringer et al. 2007).

\footnotetext{
2 We compared the interstellar hydrogen column density compilation by Dickey \& Lockman (1990) with the more recent data set of the Bonn-Leiden-Argentine $21 \mathrm{~cm}$ survey (Kalberla et al. 2005), and found that the differences relevant for us are at most one percent. Because our survey was constructed with a flux cut based on the Dickey \& Lockman results, we decided to keep the older hydrogen column density values for consistency.

$3 r_{500}$ is the radius where the average mass density inside reaches a value of 500 times the critical density of the Universe at the epoch of observation.
} 
The survey selection function determined as a function of the sky position and as a function of redshift, an important ingredient for our study, is documented in Böhringer et al. (2013) for REFLEX II and Böhringer et al. (2017) for NORAS II, where numerical data are also provided in the online material.

\section{Methods}

For the construction of the SCs from the cluster sample we used a friends-of-friends method. This can be applied straightforwardly to a homogeneous, volume-limited survey. To account for the spatially varying survey limits in our flux-limited survey, we introduced a weighing scheme that is connected to the estimated local cluster density. The weights were calculated from an integration of the luminosity function, $\phi\left(L_{\mathrm{X}}\right)$, as

$w_{i}=\frac{\int_{L_{\mathrm{X}_{0}}}^{\infty} \phi(L) \mathrm{d} L}{\int_{L_{\mathrm{X}_{i}}}^{\infty} \phi(L) \mathrm{d} L}$,

where $L_{\mathrm{X}_{0}}$ is the nominal lower limit of the sample and $L_{\mathrm{X}_{i}}$ is the lower X-ray luminosity limit that can be reached at the sky location and redshift of the cluster. The weighting scheme then accounts for the missing cluster density if the local detection limit for the X-ray luminosity is higher than $L_{\mathrm{X}_{0}}$.

In the friends-of-friends method we adopt a minimum linking length, $l_{0}$, when the nominal luminosity limit is reached. We adjust the linking length $l_{i}=l_{0} \times\left(w_{i}\right)^{1 / 3}$ if $L_{\mathrm{X}_{i}}$ is higher than $L_{\mathrm{X}_{0}}$. Since the linking length is calculated for each cluster at its location, we take the average $l_{i}$ as the linking length between the two clusters in the linking process. To introduce a higher weight for the shorter linking length, we average in the following way: $\langle l\rangle=l_{0} \cdot\left(2 /\left(1 / w_{1}+1 / w_{2}\right)\right)^{1 / 3}$. In our study the linking length can increase by up to about $40 \%$ in the extragalactic sky, by up to $70 \%$ in the ZoA.

For the main analysis we used a minimum linking length of $l_{0}=19 \mathrm{Mpc}$. This corresponds roughly to an overdensity ratio, $R_{\mathrm{Cl}}=n_{\mathrm{Cl}} /\left\langle n_{\mathrm{Cl}}\right\rangle$, of about a factor of 2 compared to the mean density of clusters in the local Universe. This value is much lower than the value of 7.8 needed for mass concentrations to collapse in the future in a $\Lambda \mathrm{CDM}$ Universe (see the definition of superstes-clusters in Chon et al. 2015); nevertheless, these structures are highly non-linear density perturbations.

We adopted a lower X-ray luminosity limit of $L_{\mathrm{X}_{0}}=$ $10^{42} \mathrm{erg} \mathrm{s}^{-1}$. With this value for $L_{\mathrm{X}_{0}}$ the survey is volume limited in most of the sky out to a redshift of $z=0.016$. The luminosity limit corresponds to a cluster mass limit of about $2.1 \times 10^{13} M_{\odot}$. We therefore included less massive galaxy groups in our study. They are definitely gravitationally bound entities, as shown by their extended X-ray emission, but they are optically often characterised by a giant elliptical galaxy surrounded by a few smaller galaxies, which in its extreme is called a 'fossil group'. Therefore, these systems are often inconspicuous in optical surveys and would not easily be recognised as galaxy groups, and are not included in the characterisation of SCs. We note that this constitutes a difference to optical studies where SC are constructed from prominent galaxy concentrations. On the other hand, they may also include some galaxy concentrations that are not so tightly bound, and are therefore not X-ray luminous. Our study compares more closely to an analysis of cosmological $N$-body simulations where groups and clusters are characterised as gravitationally bound dark matter halos.

Our analysis differs in another aspect from the construction of SC on the basis of the galaxy distribution. Galaxies tend to concentrate not only in clusters, but also in connecting filaments. Through these filamentary bridges galaxies can more easily link large structures together, while in our survey groups are only found to trace the most massive elongated structures. These differences should be kept in mind in the comparison to other studies.

\section{Results}

Constructing SCs in the local Universe $(z \leq 0.03)$ in the region around the Perseus-Pisces SC with a minimum linking length of $l_{0}=19 \mathrm{Mpc}$, we find 20 CLASSIX members defining the Perseus-Pisces SC. If we inspect a larger redshift interval we note that two additional clusters at redshifts $z=0.0302-0.0314$ are linked to the Perseus-Pisces SC. Decreasing the minimum linking length $l_{0}$ to $16 \mathrm{Mpc}$ we can define a core of the PerseusPisces SC which contains 13 cluster members. For the SGW we find seven CLASSIX cluster members. The member clusters and groups of the two SCs together with their properties are listed in Tables 1 and 2, respectively. Appendices A.1 and A.5 provide optical images with X-ray surface brightness contours of all the groups and clusters of the Perseus-Pisces SC and SGW.

\subsection{Sky distribution}

Figure 1 shows the Perseus-Pisces SC and the Southern Great Wall as they appear on the sky. The figure also shows the band of the Milky Way ( $b_{\mathrm{II}}= \pm 20^{\circ}$, dotted lines), the zone of highest Galactic absorption (blue lines), and a region of $\pm 20^{\circ}$ around the Supergalactic plane (red lines). The three clusters at redshifts $z=0.025-0.03$ and the two clusters at $z>0.03$ are indicated by squares in the image. The Perseus-Pisces SC extends over about $75^{\circ}$ on the sky. We note that it stretches in the east across the ZoA. In Fig. 2, which shows a magnification of the SC region, we have labelled some of the prominent clusters identified in the figure caption. We see that it is the cluster 3C 129 (number 7), close to the Galactic equator, that helps to bridge the SC across the ZoA. While the average linking length for the members of the Perseus-Pisces SC is $\sim 23 \mathrm{Mpc}$, after weight correction, the largest linking length due to high interstellar absorption is $\sim 30 \mathrm{Mpc}$, which bridges 3C 129 to the galaxy group in the far east, UGC 03355 (number 8).

In the earlier optical surveys mentioned in the introduction the Perseus-Pisces SC ends in the east with the Perseus cluster (number 1), since further to the east the increased extinction blocks the view. X-ray observations allow a better view into this region, but since even our survey is incomplete in the ZoA (and incompleteness has not been accounted for in the linking length), there may be more SC members hidden there.

Decreasing the minimum linking length to $16 \mathrm{Mpc}$ we find a denser core of the Perseus-Pisces SC with 13 members. These clusters are marked in the upper panel of Fig. 1 with encircled solid dots. The clusters belonging to the core are flagged in Table 1. Table 3 provides properties of the different selected structures of the Perseus-Pisces SC.

In Fig. 2 we indicate the estimated masses of the SC members by the size of the symbols, with diameter scaling with the cube root of the estimated mass, which is the best compromise to display the mass range while minimising projection effects. Even so, two clusters are hidden in projection (as noted in the figure caption). The mass estimates were obtained by means of the scaling relation of X-ray luminosity and cluster mass, which has been derived from detailed XMM-Newton X-ray observations of a representative sample of CLASSIX clusters (REXCESS), and 
Table 1. Galaxy group and cluster members of the Perseus-Pisces SC.

\begin{tabular}{|c|c|c|c|c|c|c|c|c|c|c|c|}
\hline Name & RA & Dec & Redshift & Flux & Err. & $L_{\mathrm{X}}$ & $m_{200}$ & $r_{\text {out }}$ & $n_{\mathrm{H}}$ & $R$ & Alt. name \\
\hline RXCJ2332.5+2355 & 353.1303 & 23.9266 & 0.0173 & 1.6108 & 17.50 & 0.0143 & 0.266 & 7.5 & 4.4 & $D$ & UGC 12655 \\
\hline RXCJ0015.5+1720 & 3.8764 & 17.3350 & 0.0181 & 1.9179 & 35.80 & 0.0181 & 0.308 & 8.0 & 4.0 & $D$ & NGC 57 \\
\hline RXCJ0018.3+3003 & 4.5984 & 30.0630 & 0.0223 & 2.5900 & 20.10 & 0.0374 & 0.484 & 7.5 & 5.5 & $D$ & NGC 71 \\
\hline RXCJ0021.0+2216 & 5.2628 & 22.2754 & 0.0193 & 5.1874 & 25.50 & 0.0464 & 0.554 & 16.0 & 4.2 & $D$ & SRGb063 ${ }^{(a)}$, PPS $62^{(b)}$ \\
\hline RXCJ0107.2+3224 & 16.8206 & 32.4067 & 0.0174 & 6.6932 & 10.30 & 0.0512 & 0.590 & 14.5 & 5.2 & $C$ & NGC 383 \\
\hline RXCJ0110.9+3308 & 17.7425 & 33.1486 & 0.0177 & 2.2140 & 20.00 & 0.0277 & 0.404 & 5.0 & 5.9 & $C$ & NGC 410 \\
\hline RXCJ0123.1+3327 & 20.7971 & 33.4612 & 0.0147 & 6.0909 & 18.00 & 0.0416 & 0.519 & 9.0 & 5.3 & $C$ & NGC 499 \\
\hline RXCJ0123.6+3315 & 20.9041 & 33.2516 & 0.0166 & 17.1433 & 15.00 & 0.1523 & 1.159 & 10.0 & 5.3 & $C$ & NGC 507 \\
\hline RXCJ0152.7+3609 & 28.1942 & 36.1509 & 0.0161 & 71.6749 & 4.50 & 0.4227 & 2.185 & 38.0 & 5.5 & $C$ & A 262 \\
\hline RXCJ0200.2+3126 & 30.0704 & 31.4346 & 0.0167 & 4.0825 & 16.00 & 0.0308 & 0.430 & 11.0 & 5.5 & $C$ & NGC 777 \\
\hline RXCJ0222.7+4301 & 35.6965 & 43.0182 & 0.0212 & 7.0947 & 20.80 & 0.0910 & 0.841 & 9.0 & 9.1 & $C$ & UGC 1841 \\
\hline RXCJ0238.7+4138 & 39.6977 & 41.6478 & 0.0153 & 2.3705 & 21.20 & 0.0130 & 0.253 & 14.5 & 7.6 & $C$ & NGC 996 \\
\hline RXCJ0249.5+4658 & 42.3899 & 46.9768 & 0.0274 & 2.3755 & 19.40 & 0.0433 & 0.530 & 11.5 & 13.4 & $E$ & IC $0256 / 0257$ \\
\hline RXCJ0254.4+4134 & 43.6214 & 41.5764 & 0.0172 & 110.5048 & 2.20 & 0.9477 & 3.601 & 17.5 & 9.2 & $C$ & AWM7 (NGC 1129) \\
\hline RXCJ0300.7+4428 & 45.1791 & 44.4675 & 0.0302 & 41.8408 & 4.00 & 0.9475 & 3.578 & 17.5 & 14.9 & $F$ & CIZAJ0300.7+4427 \\
\hline RXCJ0309.9+4207 & 47.4981 & 42.1255 & 0.0300 & 1.4685 & 19.70 & 0.0316 & 0.434 & 11.0 & 12.7 & $E$ & UGC 2562 \\
\hline RXCJ0310.3+4250 & 47.5755 & 42.8488 & 0.0314 & 2.6345 & 19.00 & 0.0668 & 0.681 & 9.0 & 16.2 & $F$ & \\
\hline RXCJ0319.7+4130 & 49.9498 & 41.5149 & 0.0179 & 688.3633 & 0.90 & 7.7309 & 13.227 & 17.5 & 15.7 & $C$ & A 426 (Perseus) \\
\hline RXCJ0348.1+4212 & 57.0337 & 42.2145 & 0.0174 & 3.3776 & 17.20 & 0.0324 & 0.443 & 7.5 & 23.7 & $C$ & $\mathrm{MCG}+07-08-033$ \\
\hline RXCJ0421.8+3607 & 65.4689 & 36.1200 & 0.0208 & 11.6173 & 10.10 & 0.1279 & 1.038 & 14.5 & 26.2 & $C$ & UGC 3021 \\
\hline RXCJ0450.0+4501 & 72.5146 & 45.0242 & 0.0210 & 69.6988 & 5.50 & 0.8256 & 3.300 & 17.5 & 67.9 & $C$ & $3 \mathrm{C} 129$ \\
\hline RXCJ0547.2+5052 & 86.8160 & 50.8786 & 0.0263 & 9.1313 & 11.70 & 0.1490 & 1.139 & 17.0 & 17.8 & E & UGC 3355 \\
\hline
\end{tabular}

Notes. The flux is in units of $10^{-12} \mathrm{erg} \mathrm{s}^{-1} \mathrm{~cm}^{-2}$ in the $0.1-2.4 \mathrm{keV}$ band, and the error in the following column is in percent. The X-ray luminosity, $L_{\mathrm{X}}$, is in units of $10^{44} \mathrm{erg} \mathrm{s}^{-1}$ for $0.1-2.4 \mathrm{keV}$ within $r_{500} ; m_{200}$ is the cluster mass estimated from the $L_{\mathrm{X}}$-mass relation within $r_{200} ; r_{\text {out }}$ is the radius out to which the X-ray luminosity is detected in the RASS; and $n_{\mathrm{H}}$ is the interstellar column density in the line of sight in units of $10^{20} \mathrm{~cm}^{-2} . R$ indicates to which part of the Perseus-Pisces SC the member belongs: $C=$ core, $D=$ other members $z \leq 0.025, E=$ members with $z=0.025-0.03$, and $F=$ members at $z>0.03$. In the last column we provide alternative names of the systems; groups are often designated by the name of the central dominant galaxy. ${ }^{(a)}$ Group described in Mahdavi et al. (2000), ${ }^{(b)}$ system in the Perseus-Pisces Group Survey of Trasart-Battistani (1998).

Table 2. Galaxy group and cluster members of the Southern Great Wall.

\begin{tabular}{lcrcrcccrcl}
\hline \hline Name & RA & Dec & Redshift & Flux & Err. & $L_{\mathrm{X}}$ & $m_{200}$ & $r_{\text {out }}$ & $n_{\mathrm{H}}$ & Alt. name \\
\hline RXCJ0125.5+0145 & 21.3757 & 1.7623 & 0.0184 & 5.3397 & 15.00 & 0.0452 & 0.546 & 14.0 & 3.1 & NGC 533 \\
RXCJ0125.6-0124 & 21.4198 & -1.4072 & 0.0180 & 11.7510 & 14.10 & 0.0863 & 0.816 & 24.0 & 4.1 & A 194 \\
RXCJ0149.2+1303 & 27.3049 & 13.0649 & 0.0171 & 2.3451 & 20.50 & 0.0188 & 0.318 & 9.5 & 4.9 & NGC677 \\
RXCJ0156.3+0537 & 29.0924 & 5.6228 & 0.0185 & 3.0455 & 13.20 & 0.0281 & 0.407 & 10.0 & 4.3 & NGC 741 \\
RXCJ0231.9+0114 & 37.9881 & 1.2445 & 0.0218 & 2.9004 & 21.10 & 0.0326 & 0.445 & 14.5 & 2.9 & UGC 2005 \\
RXCJ0252.8-0116 & 43.2060 & -1.2741 & 0.0235 & 7.4523 & 16.20 & 0.1150 & 0.971 & 9.5 & 5.3 & NGC 1132 \\
RXCJ0257.6+0600 & 44.4038 & 6.0160 & 0.0243 & 17.1120 & 12.00 & 0.2435 & 1.546 & 18.0 & 9.3 & A 400 \\
\hline
\end{tabular}

Notes. The meaning of the columns is the same as in Table 1 .

has been parameterised as

$M_{200}=3.75 L_{\mathrm{X}, 500}^{0.62} E(z)^{-1} h_{70}^{0.242}$,

where $E(z)=H(z)^{2} / H_{0}^{2}$ (Pratt et al. 2009). The estimated masses of the clusters and groups are listed in Tables 1 and 2. We note that the mass-X-ray luminosity relation in Eq. (2) was derived for clusters with $\mathrm{X}$-ray luminosities $L_{\mathrm{X}} \geq 4 \times$ $10^{43} \mathrm{erg} \mathrm{s}^{-1}$. For the mass estimates of the lower X-ray luminosity groups we rely on an extrapolation of this relation. We expect some steepening of this relation towards lower luminosities, due to the decreasing gas mass fraction in galaxy groups. This could be compensated for by a larger luminosity fraction of the central cool cores found in most galaxy groups and by the increase in the X-ray luminosity due to the generally higher metallicities in low mass systems. The observational results for this in the literature are controversial, however. This is mostly because the mass determination in groups from X-ray observations is difficult; without extensively long observations only the central regions of groups can be studied, which is not sufficient for a precise mass estimate. Therefore, we use the extrapolation of Eq. (2). While the uncertainty of the mass estimate for systems with $L_{\mathrm{X}} \geq 4 \times 10^{43} \mathrm{erg} \mathrm{s}^{-1}$ is about $40 \%$, it can be of the order of a factor 2 for low mass systems. Apart from a small effect on the estimated cluster masses and the sum of the cluster masses for the SC, as listed in the tables of this paper, this has no further consequences for the construction of the SC, except for an unsharp mass limit in the selection function. The Perseus cluster is by far the most massive matter concentration in the PerseusPisces SC. The next two largest clusters, 3C 129 and AWM7, are about a factor of four less massive. The Southern Great Wall contains only less massive systems, where A 400 with a mass of about $1.5 \times 10^{14} M_{\odot}$ is the most massive one.

\subsection{Size and mass}

The total length of the Perseus-Pisces SC, the largest separation between two member clusters in three dimensions, is $115.7 \mathrm{Mpc}$. 

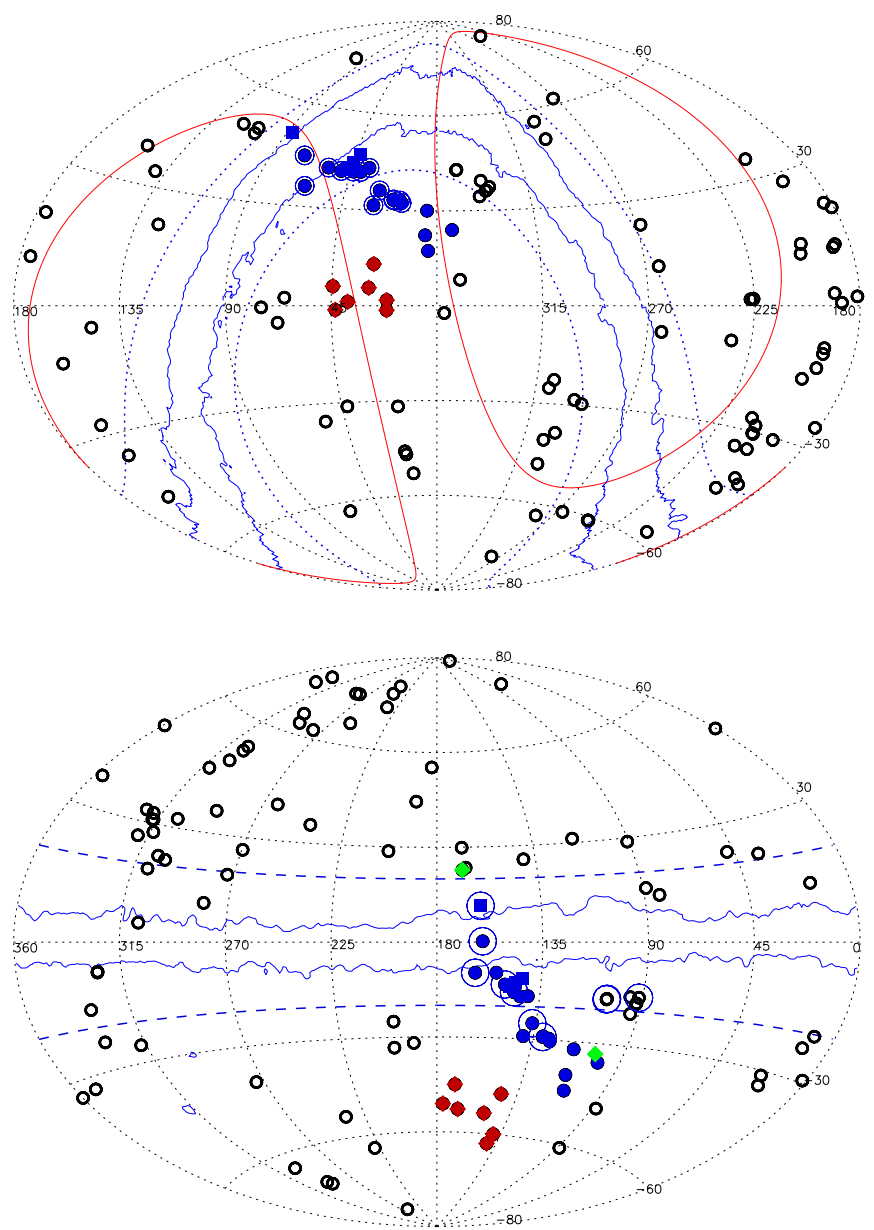

Fig. 1. Sky distribution of the CLASSIX galaxy clusters in the redshift range $z=0-0.025$. The clusters associated with the PerseusPisces SC are shown as blue solid circles $(z \leq 0.025)$ and blue solid squares $(z>0.025)$ and those belonging to the SGW as red solid diamonds. Top: map in equatorial coordinates. The members of the core part of the Perseus-Pisces SC are shown with open circles around the circles and squares. The Galactic band $\left(b_{\mathrm{II}}= \pm 20^{\circ}\right)$ is shown by the blue dotted lines, the region with high hydrogen column density $\left(n_{\mathrm{H}} \geq 2.5 \times 10^{21} \mathrm{~cm}^{-2}\right)$ is indicated by the solid blue lines, and the Supergalactic band $\left(\mathrm{SGB}= \pm 20^{\circ}\right)$ by the red lines. Bottom: map in Galactic coordinates. The larger blue open circles show the members of the Perseus-Pisces SC found with an increased lower luminosity limit of $10^{43} \mathrm{erg} \mathrm{s}^{-1}$ as explained in Sect. 4.3. The two green points show the two clusters A 569 (positive $b_{\text {II }}$ ) and A 2634 (negative $b_{\text {II }}$ ), which are part of the discussion in Sect. 5.

Apart from five member clusters, which have a redshift greater than $z=0.025$ and are labelled with squares in Figs. 1 and 2, the redshift range of the cluster members is $z=0.0147-0.0223$, which corresponds to a radial extent of $32.3 \mathrm{Mpc}$ (including the five clusters the maximum extent is $71.2 \mathrm{Mpc}$ ). Thus, most of the Perseus-Pisces SC is oriented closely along the plane of the sky. For an approximate volume estimate of the total SC, we take the sum of the spheres with radius $19 \mathrm{Mpc}$ (equal to the minimum linking length) around all member clusters accounting for the overlap, and obtain a value of $2.9 \times 10^{5} \mathrm{Mpc}^{3}$. Table 3 lists these properties for three different parts of the Perseus-Pisces SC $\left(\mathrm{PP}=\right.$ full $\mathrm{SC}, \mathrm{PP}_{\text {in }}=$ structure at $z \leq 0.03, \mathrm{PP}_{\text {core }}=$ core region with 13 members) and for the SGW.

Using this value for the volume, we can derive a mass estimate for the SC. For 22 cluster members we find a clus-

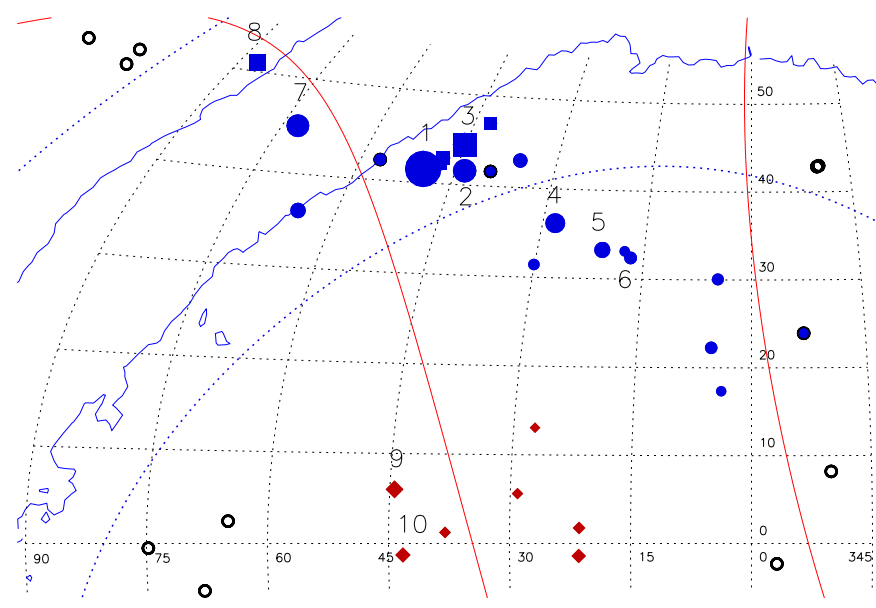

Fig. 2. Perseus-Pisces SC (blue filled circles $[z \leq 0.025]$ and squares $[z>0.025])$ and Southern Great Wall (red diamonds). The size of the symbols indicates the estimated mass of the clusters, as described in the text. The open symbols indicate clusters that are not members of these SCs. Some massive galaxy clusters and groups are labelled: (1) Perseus cluster, (2) AWM7, (3) CIZAJ0300.7+4427, (4) A 262, (5) NGC 499 and NGC 507 (here blended together), (6) NGC 383, (7) 3C 129, (8) UGC 3355, (9) A 400, (10) NGC 1132. The group UGC 2562 is hidden behind the Perseus cluster. The lines marking the region of high interstellar hydrogen column density, the ZoA, and the Supergalactic band are the same as in Fig. 1.

ter density of $7.6 \times 10^{-5} \mathrm{Mpc}^{-3}$ (with weights the density is $2.0 \times 10^{-4} \mathrm{Mpc}^{-3}$ ), which is about a factor of 2.8 higher than the mean density of clusters with $L_{\mathrm{X}}>L_{\mathrm{X}_{0}}$ of $7.2 \times 10^{-5} \mathrm{Mpc}^{-3}$, determined from the mean luminosity function in a volume up to $z=0.4$ (Böhringer et al. 2014). A cluster overdensity ratio of $R_{\mathrm{cl}}=2.8$ corresponds to a cluster overdensity $\Delta_{\mathrm{cl}}=$ $\left(n_{\mathrm{cl}}-\left\langle n_{\mathrm{cl}}\right\rangle\right) /\left\langle n_{\mathrm{cl}}\right\rangle=1.8$. This can be related to the overdensity of matter by means of the predicted value for the large-scale structure bias, which depends on the lower mass limit of the cluster sample. From theoretical calculations (Tinker et al. 2010) based on simulations and from our previous studies of clusters in the REFLEX sample, we find a value for the cluster bias around 1.6 for the mass range involved here (for details of these considerations see Böhringer et al. 2020). Therefore we get an overdensity for the total matter of $\Delta_{\mathrm{DM}} \sim 1.1$ and a matter overdensity ratio of $R_{\mathrm{DM}} \sim 2.1$. Together with the SC volume derived above, this leads to a SC mass estimate of $2.5 \times 10^{16} M_{\odot}$. It can be compared to the sum of the mass of all clusters in the Perseus-Pisces SC of $3.6 \times 10^{15} M_{\odot}$, which is only a small fraction of the SC mass. We can also compare this number to the early estimate by Joeveer et al. (1978) with a value of $2 \times 10^{16} h_{50}^{-1} M_{\odot}$, which is of a similar order of magnitude. Taking into account that our $\mathrm{SC}$ stretches into the ZoA, and is thus larger, and that we have been quite generous in staking out the SC volume, the two numbers are surprisingly consistent. For smaller parts of the PerseusPisces SC similar calculations can be performed and the results on the estimated SC mass are provided in Table 3.

The SGW merges with the Perseus-Pisces SC if we choose a minimum linking length of 21 instead of $19 \mathrm{Mpc}$. The shortest distance between members of each SC is $29.8 \mathrm{Mpc}$. Thus, these are the nearest superclusters to each other in the local Universe.

The largest extent between two members in three dimensions in the SGW is $45.8 \mathrm{Mpc}$ and the volume for a spherical zone around each cluster with $19 \mathrm{Mpc}$ radius is $1.2 \times 10^{5} \mathrm{Mpc}^{3}$, which yields a weighed cluster density of $1.2 \times 10^{-4} \mathrm{Mpc}^{-3}$, similar to the Perseus-Pisces SC. With the same arguments as above we 
Table 3. Properties of the Perseus-Pisces SC and the SGW constructed with a minimum linking length of $19 \mathrm{Mpc}$.

\begin{tabular}{|c|c|c|c|c|c|c|c|c|c|c|c|c|}
\hline Name & $N_{\mathrm{CL}}$ & $w N_{\mathrm{CL}}$ & $\begin{array}{r}\text { Volume } \\
\left(10^{5} \mathrm{Mpc}^{3}\right) \\
\end{array}$ & $\begin{array}{r}\text { Cl. mass } \\
\left(10^{14} M_{\odot}\right)\end{array}$ & $\begin{array}{l}\text { Est. mass } \\
\left(10^{15} M_{\odot}\right)\end{array}$ & $\begin{array}{r}\text { Length } \\
(\mathrm{Mpc}) \\
\end{array}$ & $\langle z\rangle$ & $z_{\min }$ & $z_{\max }$ & $\begin{array}{r}\text { Density } \\
\left(10^{-4} \mathrm{Mpc}^{-3}\right) \\
\end{array}$ & $R_{\mathrm{CL}}$ & $R_{\mathrm{DM}}$ \\
\hline PP & 22 & 58.3 & 2.9 & 35.8 & 24.9 & 115.7 & 0.0205 & 0.0147 & 0.0314 & 2.0 & 2.8 & 2.1 \\
\hline $\mathrm{PP}_{\text {in }}$ & 20 & 49.0 & 2.8 & 31.6 & 21.5 & 115.7 & 0.0195 & 0.0147 & 0.0300 & 1.8 & 2.4 & 1.9 \\
\hline $\mathrm{PP}_{\text {core }}$ & 13 & 30.4 & 1.5 & 27.8 & 12.9 & 63.4 & 0.0176 & 0.0147 & 0.0210 & 2.1 & 2.9 & 2.2 \\
\hline SGW & 7 & 14.6 & 1.1 & 5.0 & 6.8 & 45.8 & 0.0202 & 0.0171 & 0.0243 & 1.4 & 1.9 & 1.6 \\
\hline
\end{tabular}

Notes. For the Perseus-Pisces SC we provide values for three different extensions: $\mathrm{PP}=$ full $\mathrm{SC}, \mathrm{PP}_{\text {in }}=$ structure at $z \leq 0.03$, and $\mathrm{PP}$ core $=$ core region. The columns give (from left to right) $N_{\mathrm{CL}}$ and $w N_{\mathrm{CL}}$ (the number of members and the weighted number of members, respectively); the SC volume; cl. mass (the sum of the estimated masses of the member clusters); est. mass and length (the estimated SC mass and its extent); $\langle z\rangle, z_{\mathrm{min}}$, and $z_{\max }$ (the mean, minimum, and maximum redshift); the number density of clusters in the $\mathrm{SC} ; R_{\mathrm{CL}}$ and $R_{\mathrm{DM}}$ (the overdensity ratio of the cluster number density and matter density in the SC compared to the cosmic mean, respectively).

arrive at a mass estimate for the SGW of $6.8 \times 10^{15} M_{\odot}$. The sum of the estimated masses of the individual clusters, with a value of $5.0 \times 10^{14} M_{\odot}$, constitutes a fraction of $\sim 7.3 \%$, which is smaller by about a factor of two than the value for Perseus-Pisces. This is due to the fact that the SGW contains no massive clusters. The SC properties are also summarised in Table 3.

\subsection{Alternative linking schemes}

In a subsequent paper of this series we will explore the SC construction for various lower X-ray luminosity limits. While keeping the goal of the selection of structures with an overdensity ratio of at least 2 fixed, we studied how the selection of the SCs change when restricting the cluster sample to objects with higher minimum X-ray luminosity. In general, the overall structure selection is quite robust, but in detail the linking of some extensions of the structures can change.

When we choose a higher X-ray luminosity limit for the cluster sample in this approach, we need to adjust the linking length to the more sparse cluster density if we want to keep the minimum overdensity ratio constant. Thus, on the one hand, there is a possibility to link to new X-ray luminous clusters. On the other hand, connections relying on low luminosity objects can disappear. If, for the construction of the Perseus-Pisces SC, we make an extreme change by increasing the lower X-ray luminosity limit by a factor of 10 to $10^{43} \mathrm{erg} \mathrm{s}^{-1}$, we get the change illustrated in the lower panel of Fig. 1. In the figure the groups and clusters linked by the default construction to form the PerseusPisces SC are shown by solid blue dots. The clusters selected at the high luminosity limit are marked by large blue, open circles. We note that the main chain of the Perseus-Pisces SC is traced well in both cases. However, while at low $L_{\mathrm{X}_{0}}$ there is an extension consisting of a small group of low luminosity objects in the south-west, two clusters towards the north-west are linked at high $L_{\mathrm{X}_{0}}$. The latter extension is interesting in comparison to the galaxy distribution, as discussed in Sect. 5.

\subsection{Three dimensional structure}

As a representation of the three-dimensional structure of the Perseus-Pisces SC and the Southern Great Wall, we show their structure in Supergalactic Cartesian coordinates in three projections in Fig. 3. The members of the Perseus-Pisces SC are shown as blue filled circles, and those of the SGW as red filled squares. The five clusters of the Perseus-Pisces SC with $z>0.025$ are marked by additional embedding open diamonds. We note that they form a clearly separated group of systems, as shown by two of the projections.
With respect to the Supergalactic plane, the major axis of the Perseus-Pisces SC has an inclination of about $34^{\circ}$. Within the Supergalactic plane the SC main axis has an angle of about $36^{\circ}$ with the $X$-axis towards the positive $Y$-axis. The Perseus cluster occupies a central position in the SC. The SGW has a lager extent perpendicular to the Supergalactic plane than within the plane.

In Fig. 4 we show a three-dimensional representation of the two SC. We note that the two SCs are close but clearly separated. The distribution of groups and clusters in this figure can be compared to the three-dimensional matter distribution inferred from peculiar motions of galaxies in the local Universe as studied by the group of Tully et al. (e.g. Courtois et al. 2013; Tully et al. 2014, 2019). A good illustration for the comparison is Fig. 8 by Courtois et al. (2013), where we see clearly the mass concentrations of the Perseus-Pisces SC and the Southern Great Wall in a very similar configuration to that shown in Fig. 4.

Among the nearby SC the Perseus-Pisces SC stands out with its remarkable morphology. It features a long chain of groups and clusters in an almost straight and linear structure. On the sky the main axis of this $\mathrm{SC}$ has a position angle of $\sim 107^{\circ}$ (for details of the determination of the angle see Appendix A.3).

Earlier studies of the Perseus-Pisces SC, for example the one by Joeveer et al. (1978), have found that the shapes of the clusters are aligned with the elongation of the SC. We can test if this is also supported by X-ray observations by studying the shapes of the X-ray images of the SC members. This is explained in more detail in Appendices A.1 and A.2. In the appendices we study the alignment in two respects. First, we study the alignment of the cluster shapes with the main axis of the SC, and then we inspect whether the clusters are aligned with the direction of the tidal pull of all other cluster members. In general, we see no evidence of the two types of alignment.

A very clear alignment is found, however, in the shapes of the Perseus cluster and the neighbouring group AWM7. The ellipticity of both systems is well aligned with the separation vector of the two. The alignment, which is almost horizontal (position angle $\sim 90^{\circ}$ ), is only weakly aligned with the SC with position angle $107^{\circ}$.

\subsection{Member clusters}

In our previous studies of superstes-clusters, which have a larger overdensity, we found that the mass or X-ray luminosity distribution of the member clusters is more top heavy than the distribution of these properties of clusters in the field. Would we find the same effect in these larger structures at lower overdensity? Figure 5 shows the cumulative, normalised X-ray 

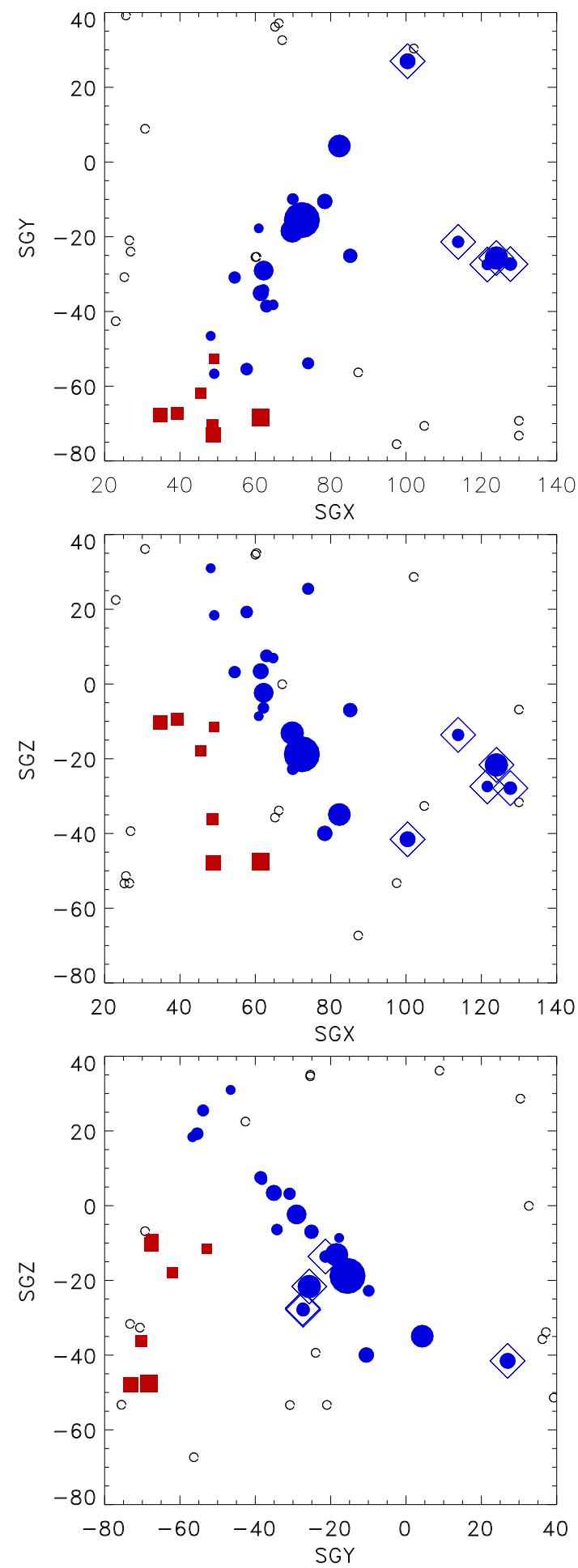

Fig. 3. Perseus-Pisces SC and Southern Great Wall in Supergalactic coordinates, where the Supergalactic plane is defined by SGX and SGY. The members of the Perseus-Pisces SC are shown by blue filled circles, and those of the Southern Great Wall by red squares.

luminosity distribution of the groups and clusters in the PerseusPisces SC compared to that of clusters in the surrounding volume at $z \leq 0.03$ (also not contained in the Southern Great Wall). We see an interesting trend, that the Perseus-Pisces SC contains more luminous clusters. A Kolmogorov-Smirnov test shows, however, that this result $(77 \%$ probability that the two distributions agree) based on just one SC is not significant.

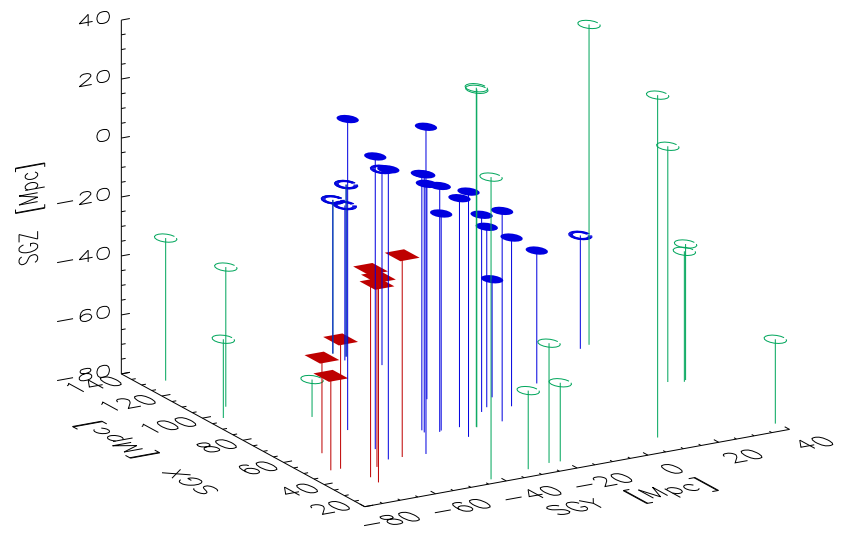

Fig. 4. Three-dimensional representation of the CLASSIX cluster distribution in a box of $120 \mathrm{Mpc}$ side length around the Perseus-Pisces SC in Supergalactic coordinates as indicated on the axes of the plot. The members of the Perseus-Pisces SC are shown by filled blue circles and those of the Southern Great Wall by filled red diamonds, while all other clusters are marked by open circles.

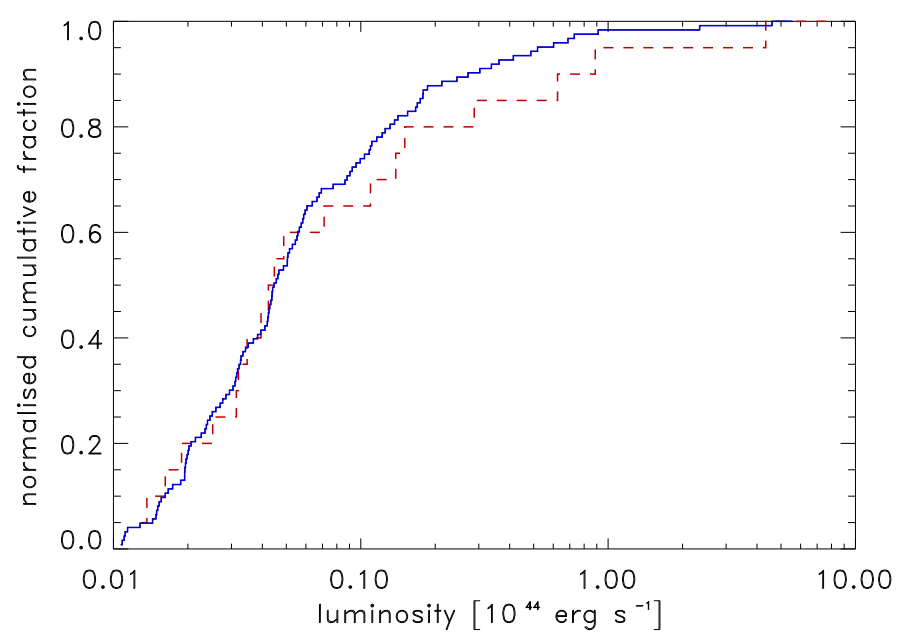

Fig. 5. Cumulative normalised distribution of the X-ray luminosity of the groups and clusters of galaxies in the Perseus-Pisces SC (at $z \leq 0.03$ ) (red dashed line) compared to the luminosity distribution of the systems outside the Perseus-Pisces SC and the Southern Great Wall (blue solid line).

\section{Discussion}

We took a new approach to characterise the Perseus-Pisces SC and the Southern Great Wall with X-ray luminous clusters. In addition to finding a very similar structure of the SC to that found in previous optical studies, we now see that it extends through the ZoA. This makes the Perseus-Pisces SC the largest SC in the local Universe at $z \leq 0.03$, with more groups and cluster members than even the Southern Great Wall. It also makes the largest contribution to the flattened local superstructure extending the Local Supercluster to a radius of about $100 \mathrm{Mpc}$, which we studied previously (Böhringer et al. 2021).

Based on radio observations of neutral hydrogen, the galaxy distribution in the surroundings of the Perseus-Pisces SC was studied, extending the redshift survey into the ZoA. Hauschildt (1987) and Chamaraux et al. (1990) investigated an extension of the Perseus-Pisces SC across the ZoA, testing an earlier suggestion by Burns \& Owen (1979) of an extension up to the cluster A 569 (see Fig. 1, bottom). Both redshift surveys find a set of galaxies around 3C 129 connecting it to the Perseus-Pisces SC. 
While Hauschildt (1987) argues that the SC seems to end there, Chamaraux et al. (1990) find some more redshifts indicating a connection to A 569. Ramatsoku et al. (2016) find a larger number of galaxies in a halo around 3C 129 at the same distance as the Perseus-Pisces SC, further strengthening this connection. As shown above, we find in our study that the Perseus-Pisces SC extends through the cluster, 3C 129 close to the galactic equator, to the other side of the ZoA to UGC 3355. A 569 has 3C 129 as its closest neighbour with a distance of $36.2 \mathrm{Mpc}$, and is thus too far away for the given linking length.

An additional cluster discussed in these previous works as a member of the Perseus-Pisces SC located at the west side of the $\mathrm{SC}$ is A 2534 (see Fig. 1, bottom). This cluster is even further away from the nearest SC member with a distance of $41.8 \mathrm{Mpc}$. While it appears in Fig. 1 near to a small western group of SC members, its higher redshift of $z=0.0312$ is responsible for the distance and it is not linked to the SC, even if we relax the condition for members to be at $z \leq 0.03$.

Another interesting point is a comparison to the galaxy distribution characterising the Perseus-Pisces SC including many redshifts in the ZoA from the work by Kraan-Korteweg et al. (2018). They show (in their Fig. 10) a very prominent filament of galaxies roughly following the chain of galaxy groups and clusters from UGC 3355 in the east to UGC 12655 in the west. This galaxy filament then continues by turning north and crossing the ZoA roughly at galactic longitude $90^{\circ}$. Even though this extension of the galaxy filament is also prominent, it is not traced by a chain of groups and clusters. However, approximately in the middle of this extension we find a group of X-ray luminous systems, which indicate at least one location in this filament. Two of these clusters are luminous enough that they are linked to the Perseus-Pisces SC with a higher low luminosity cut and corresponding to a larger linking length, as explained in Sect. 4.3. This seems to be the only indication marking the western upturn of the SC as traced by clusters.

Characterising the shapes of the groups and clusters in the Perseus-Pisces SC as traced by the X-ray emission, we find no significant alignment of cluster elongations with the main axis of the SC, except for the effect that Perseus and AWM7 are pointing to each other. This is different from earlier findings using optical surveys. A major reason for this could be that the X-ray emission is focussed on the central, virialised, and thermalised part of the clusters, which by nature has acquired a rounder shape. This effect is further strengthened as the X-ray emission follows roughly equipotential contours that are rounder than the galaxy and dark matter distribution. In the study by Buote \& Canizares (1996) one can typically find, for example, ellipticity values that are three times higher in the projected dark matter distribution compared to the X-ray surface brightness. Therefore, even though X-rays are a very sensitive probe of the cluster structure, they are not particularly able to capture the large-scale ellipticity of a cluster. It would be interesting in this context to study the shape of the member clusters of the Perseus-Pisces SC in the optical separating the tracer galaxies into a red and blue or early- and late-type population. In our previous studies (e.g. Schindler et al. 1999, for the Virgo cluster, and Braglia et al. 2009), we find that the red early-type galaxy population follows the X-ray emission very closely, whereas the blue galaxies preferentially populate the outskirts. Therefore, we would expect that alignment effects with the SC would most strongly show up in the blue galaxy population.

Another difference between our work and studies based on optical surveys of the galaxy population is the inclusion of compact groups with one dominant giant elliptical (some of them qualify as fossil groups), which would be very inconspicuous in the optical. However, this choice is closest to choosing dark matter halos with a given mass limit with the mass measured inside a certain overdensity radius, which in our case is $r_{500}$. One of the most compact of the groups in our sample is NGC 410. As an example we give more details on this object in Appendix A.3. The X-ray emission of the group can be resolved with $X M M$-Newton. Fitting a $\beta$-model, we find a core radius of the $X$-ray image of $\sim 5$ arcsec. The group was also studied by O'Sullivan et al. (2017) who confirm that the X-ray emission of the group is from a thermal intracluster plasma with a temperature of $\sim 0.98 \mathrm{keV}$. We can trace the X-ray emission in the XMMNewton observation out to about $150 \operatorname{arcsec}(\sim 54 \mathrm{kpc})$, whereas the estimate for $r_{200}$ is about $419 \mathrm{kpc}$. Thus, for some of these very compact groups we observe only the inner core of the system. This is also one more reason why it is difficult to detect the large-scale structure alignment for these low mass systems in X-rays.

On the other hand, two groups and clusters have been associated with the Perseus-Pisces SC by Joeveer et al. (1978), A 347 and NGC 315, which are not X-ray luminous enough to be included in our study. A 347 was also assigned to the PerseusPisces SC by Gregory et al. (1981). In these two objects we observe no X-ray emission in the RASS for A 347, for which we can set an upper limit on the X-ray luminosity of $L_{\mathrm{X}}<$ $0.9 \times 10^{42} \mathrm{erg} \mathrm{s}^{-1}$, and for NGC 315 we find a faint X-ray source with an upper limit of $L_{X}<0.8 \times 10^{42} \mathrm{erg} \mathrm{s}^{-1}$. Since X-ray observations probe the relaxed core of clusters, the non- or faint detections of the two poor clusters imply masses for their relaxed parts of $m_{200} \leq 2 \times 10^{13} M_{\odot}$.

\section{Summary and conclusion}

One of the major goals of this paper is to provide a detailed reference for the structure and properties of the Perseus-Pisces $\mathrm{SC}$ and the neighbouring Southern Great Wall as traced by Xray luminous clusters. We find that the Perseus-Pisces SC with a length of $\sim 116 \mathrm{Mpc}$ and a mass of the order of $2.5 \times 10^{16} M_{\odot}$ is the largest SC in the local Universe out to a redshift of $\sim 0.03$. In our study we assign 22 groups and clusters with a total mass of about $3.6 \times 10^{15} M_{\odot}$ to this SC (including 2 clusters at $z=0.03-0.0314$ ). The SGW is about two and a half times smaller in length and three times smaller in mass and number of members.

The two SCs defined in this work have a cluster density that is larger by a factor of $\sim 2.5$ compared to the mean density in the study volume, which implies a matter overdensity ratio of about 2. Thus, both structures are well within the non-linear regime, which also explains their highly elongated shapes. However, their overdensity is not nearly high enough for these structures to collapse to one unit in the future in a $\Lambda$ CDM universe. Instead, they will fragment and form a series of very massive clusters in the future.

The Perseus-Pisces SC is a remarkable straight chain of galaxy groups and clusters, which extends even further in one direction than previously inferred from optical surveys, because we can now trace it through the ZoA. Previous neutral hydrogen surveys had already given indications of this. The SC is dominated by the Perseus cluster, while the Southern Great Wall is not inhabited by massive clusters.

The shapes of the X-ray images of the Perseus-Pisces SC members are not found to align with the major axis of the SC. The Perseus cluster and AWM7 are, however, well aligned with each other. 
Acknowledgements. We thank the referee for helpful comments. HB and GC acknowledge support of the Deutsche Forschungsgemeinschaft through the Munich Excellence Cluster "Universe". G.C. acknowledges support by the DLR under grant no. 50 OR 1905.

\section{References}

Bahcall, N. 1988, ARA\&A, 26, 631

Balaguera-Antolinez, A., Sanchez, A., Böhringer, H., et al. 2011, MNRAS, 413, 386

Balaguera-Antolinez, A., Sanchez, A., Böhringer, H., et al. 2012, MNRAS, 425, 2244

Bardeen, J. M., Bond, J. R., Kaiser, N., et al. 1986, ApJ, 304, 15

Batuski, D. J., \& Burns, J. O. 1985, ApJ, 299, 5

Böhringer, H., Schuecker, P., Guzzo, L., et al. 2004, A\&A, 425, 367

Böhringer, H., Schuecker, P., Pratt, G. W., et al. 2007, A\&A, 469, 363

Böhringer, H., Chon, G., Collins, C. A., et al. 2013, A\&A, 555, A30

Böhringer, H., Chon, G., Collins, C. A., et al. 2014, A\&A, 570, A31

Böhringer, H., Chon, G., Bristow, M., et al. 2015, A\&A, 574, A26

Böhringer, H., Chon, G., Retzlaff, J., et al. 2017, AJ, 153, 220

Böhringer, H., Chon, G., \& Collins, C. A. 2020, A\&A, 633, 19

Böhringer, H., Chon, G., \& Trümper, J. 2021, A\&A, 651, A15

Braglia, F. G., Pierini, D., Biviano, A., et al. 2009, A\&A, 500, 947

Buote, D. A., \& Canizares, C. R. 1996, ApJ, 457, 565

Burns, J. O., \& Owen, F. N. 1979, AJ, 84, 1478

Chamaraux, P., Cayatte, V., Balkoski, C., et al. 1990, A\&A, 229, 340

Chincarini, G. L., Giovanelli, R., \& Haynes, M. P. 1983, A\&A, 121, 5

Chon, G., \& Böhringer, H. 2013, MNRAS, 429, 3272

Chon, G., Böhringer, H., Collins, C. A., et al. 2014, A\&A, 567, A144

Chon, G., Böhringer, H., \& Zaroubi, S. 2015, A\&A, 575, L14

Collins, C. A., Guzzo, L., Böhringer, H., et al. 2000, MNRAS, 319, 939

Courtois, H. M., Pomàrede, D., Tully, R. B., et al. 2013, AJ, 146, 69 de Vaucouleurs, G., de Vaucouleurs, A., \& Corwin, H. G., Jr., et al. 1991, The Third Catalogue of Bright Galaxies (RC3) (Austin: University of Texas Press) Dickey, J. M., \& Lockman, F. J. 1990, ARA\&A, 28, 215

Einasto, J., Einasto, M., Tago, E., et al. 2007, A\&A, 462, 811

Gregory, S. A., Thompson, L. A., \& Tifft, W. G. 1981, ApJ, 243, 411

Hauschildt, M. 1987, A\&A, 184, 43

Joeveer, M., \& Einasto, J. 1978, IAU Symp., 79, 241

Joeveer, M., Einasto, J., \& Tago, E. 1978, MNRAS, 185, 357

Kaiser, N. 1986, MNRAS, 222, 323

Kalberla, P. M. W., Burton, W. B., \& Hartmann, D. 2005, A\&A, 440, 775

Kerscher, M., Mecke, K., Schuecker, P., et al. 2001, A\&A, 377, 1

Kraan-Korteweg, R. C., van Driel, W., Schröder, A. C., et al. 2018, MNRAS, 481, 1262

Lahav, O., Santiago, B. X., Webster, A. M., et al. 2000, MNRAS, 312, 166

Mahdavi, A., Böhringer, H., Geller, M. J., et al. 2000, ApJ, 534, 114

Mo, H. J., \& White, S. D. M. 1996, MNRAS, 282, 347

O'Sullivan, E., Ponman, T. J., Kolokythas, K., et al. 2017, MNRAS, 472, 1482

Pratt, G. W., Croston, J. H., Arnaud, M., \& Böhringer, H. 2009, A\&A, 498, 361

Ramatsoku, M., Verheijen, M. A. W., Kraan-Korteweg, R. C., et al. 2016, MNRAS, 460, 923

Schindler, S., Binggeli, B., \& Böhringer, H. 1999, A\&A, 343, 420

Schuecker, P., Böhringer, H., Guzzo, L., et al. 2001, A\&A, 368, 86

Schuecker, P., Guzzo, L., Collins, C. A., et al. 2002, MNRAS, 335, 807

Schuecker, P., Böhringer, H., Collins, C. A., et al. 2003a, A\&A, 398, 867

Schuecker, P., Caldwell, R. R., Böhringer, H., et al. 2003b, A\&A, 402, 53

Sheth, R. K., \& Tormen, G. 1999, MNRAS, 308, 119

Tinker, J. L., Robertson, B. E., Kravtsov, A. V., et al. 2010, ApJ, 724, 878

Trasart-Battistani, R. 1998, A\&AS, 130, 341

Trümper, J. 1993, Science, 260, 1769

Tully, R. B., Courtois, H., Hoffman, Y., et al. 2014, Nature, 513, 71

Tully, R. B., Pomarede, D., Graziani, R., et al. 2019, ApJ, 880, 24

Voges, W., Aschenbach, B., Boller, T., et al. 1999, A\&A, 349, 389

Wen, Z. L., Han, J. L., \& Liu, F. S. 2009, ApJS, 183, 197 


\section{Appendix A: Additional material}

\section{A.1. X-ray - Optical images of the Perseus-Pisces supercluster members}

Figures A.1-A.4 provide images of the member groups and clusters of the Perseus-Pisces SC. They consist of X-ray surface brightness contours superposed on optical images from the DSS digitisation of the photographic sky surveys. The X-ray contours are based on XMM-Newton observations when available. In this case all three detectors were combined with a scaling of the pnimages with a factor of 3.3 with respect to the MOS images. For systems with no XMM-Newton observations, we use X-ray images from the ROSAT All-Sky Survey. An exception is the Perseus cluster, where the field of view of XMM-Newton is too small to show the entire cluster in one pointing. For this reason, we use a ROSAT pointed observation for the Perseus cluster.

Overplotted we show the direction of the main axis of the Perseus-Pisces SC and the direction of the combined tidal forces of all other SC members on the clusters for comparison with the cluster shapes. For the RASS images we do not show these directions, as the photon statistics are too poor for a reliable determination of the cluster or group elongation. Appendix A.2 provides details of how these directions were determined.

\section{A.2. Alignment of galaxy clusters with the supercluster}

To investigate whether the cluster shapes in the Perseus-Pisces $\mathrm{SC}$ are aligned with the SC elongation, we determined the main axis of the SC by deriving the tensor of the moment of inertia and its principle axis in projection onto the sky. If we determine the major axis of the inertia tensor with just the object distribution or with the objects weighed by their mass, we obtain in both cases a position angle for the major axis of about $\mathrm{PA} \sim 107^{\circ}$.

To see whether the possible orientation of the cluster shapes follows the tidal forces of the SC members, we also determined the moment of inertia and its major axis for each cluster position scaling the distance to each other cluster in a special way. For the conventional moment of inertia the mass points introduce a weight that is proportional to the square of the distance, while the gravitational force depends on the inverse of the distance squared. Therefore, we scale every SC member with its mass and with an additional factor of the distance to the inverse fourth power, $\Delta^{-4}$, in the tensor of the moment of inertia.

The results of both calculations are illustrated in Fig. A.5, where we show the major axis of the Perseus-Pisces SC as a whole by the dotted line and the direction of the tidal forces for each SC member by short solid lines. Both directions are displayed in the images of those Perseus-Pisces SC members in Figs. A.1-A.4, which have deeper pointed observations by $X M M-N e w t o n$ or ROSAT. As discussed in the main text, we see no significant evidence of an alignment of the cluster shapes with the SC. Both Perseus and AWM7 experience mutual tidal forces without much disturbance by the other SC members, as can be seen in Fig. A.5. Here we see that the cluster shapes follow the direction of the tidal forces very closely.

\section{A.3. Identification of $R X C J 0021.0+2216$}

One of the groups associated with the Perseus-Pisces SC, RXCJ0021.0+2216, has a different appearance (Fig. A.1, middle right) than typically found for the other systems. The X-ray emission is extended and has a diffuse, low surface brightness. However, the X-ray emission is not peaked on a dominant elliptical galaxy, in this case NGC 80 at $z=0.01947$, which is the bright galaxy seen in the north of the image. Therefore, it is worth looking at the identification of this source in more detail. In a detailed survey of X-ray emission of galaxy groups in the CfA redshift survey by Mahdavi et al. (2000), this system was identified as the X-ray luminous group SRGb063 at $z=0.0189$. The NASA Extragalactic Database (NED) lists nine galaxies with redshifts $z=0.019 \pm 0.0013$ within a region of 7 arcmin radius around the centre of the emission, well inside the extent of the X-ray emission, consistent with this result. The group was also identified with the galaxy group found in the optical in the Perseus-Pisces Group Survey by Trasart-Battistani (1998) with the name PPS 62 and a redshift of $z=0.0188$. In the same region Wen et al. (2009) found a galaxy cluster at $z=0.1991$, which is supported by only one redshift in NED.

The extended shallow X-ray emission is more consistent with a low redshift group than with a more distant X-ray luminous galaxy cluster, which should appear more compact. The spectral X-ray hardness ratio is consistent with thermal emission from intragroup or intracluster medium. Based on the combined evidence, we therefore identify the X-ray source with the low luminosity group, which makes it a member of the Perseus SC.

\section{A.4. Nature of the X-ray emission in NGC 410}

As we discussed in the main text, some of the groups in the cluster sample appear compact. Here we show one of the most compact of these groups in X-rays, the group associated with NGC 410 (RXCJ0110.9+3308). An XMM-Newton image of the system is shown in Fig. A.1 (lower right). In Fig. A.6 we show the X-ray surface brightness profile obtained from the $X M M-N e w t o n$ observation of the group. The emission is clearly extended, as can be seen by comparison with the point spread function (PSF), also shown in the figure. A fit of a beta-model to the profile taking a convolution with the PSF into account yields a core radius of about $2 \mathrm{kpc}$. This is indeed compact compared to the estimated $r_{500}$ of $\sim 400 \mathrm{kpc}$.

O'Sullivan et al. (2017) studied this system in more detail in X-rays together with a series of other nearby galaxy groups. They found that the X-ray emission originates from a thermal plasma with a temperature of about $0.98 \mathrm{keV}$. Thus, the observed $\mathrm{X}$-ray emission is clearly consistent with an intragroup medium origin.

\section{A.5. Images of the members of the Southern Great Wall}

Figures A.7 and A.8 provide images of the seven group and cluster members of the Southern Great Wall as derived from the ROSAT All-Sky Survey overlayed as contours on DSS images. 

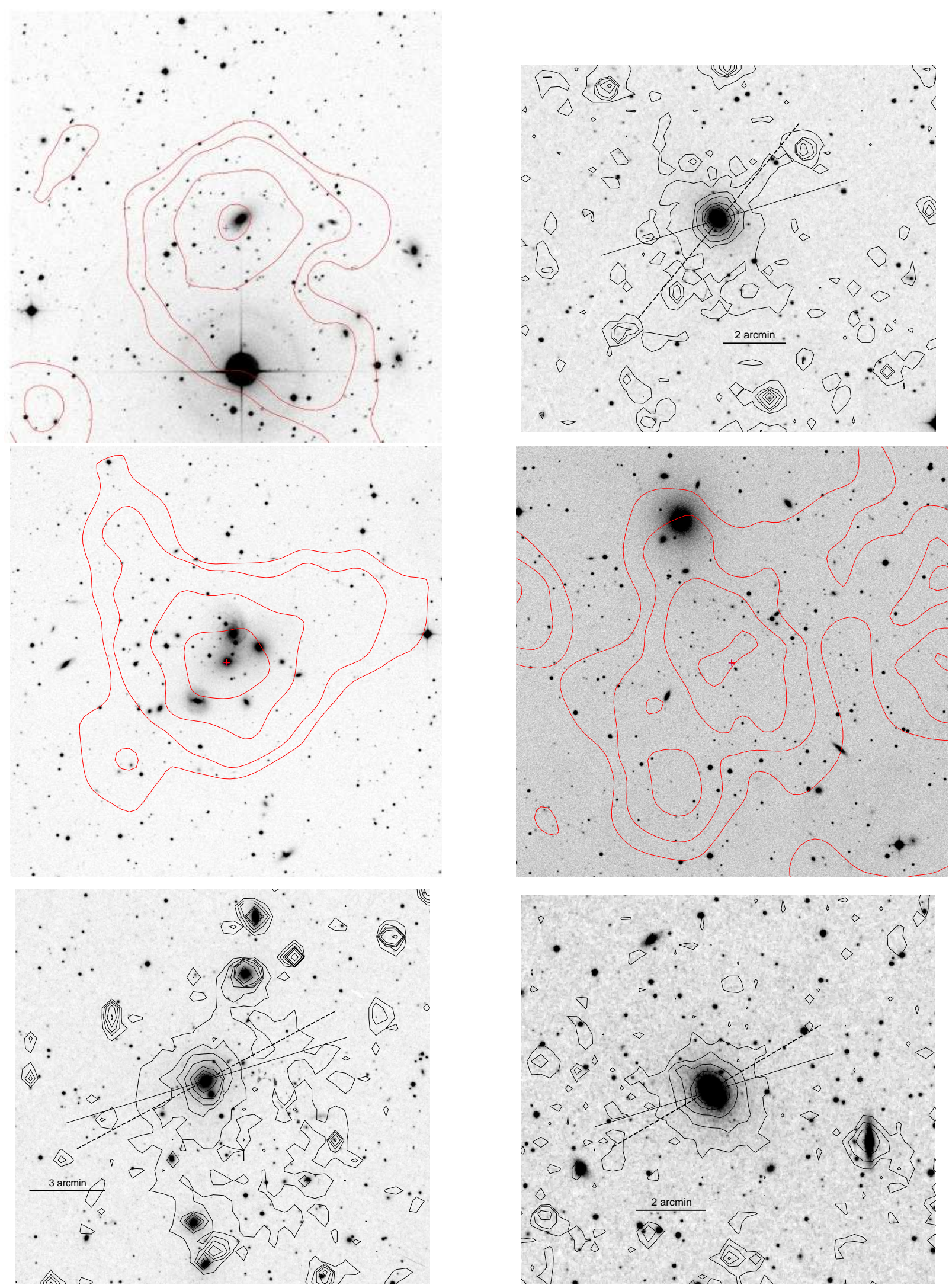

Fig. A.1. Contours of the X-ray surface brightness superposed on optical images from the DSS database. The X-ray data are from the RASS or XMM-Newton observations. All images here and in the following figures are oriented such that north is up and east to the left. Upper left: RXCJ2332.5+2355, UGC 12655, X-ray data from RASS; upper right: RXCJ0015.5+1720, NGC 57, X-ray data from XMM-Newton; middle left: RXCJ0018.3+3003, NGC 71, X-ray data from RASS; middle right: RXCJ0021.0+2216, SRGb063, PPS 62, X-ray data from RASS; lower left: RXCJ0107.2+3224, NGC 383; X-ray data from XMM-Newton; lower right: RXCJ0110.9+3308, NGC 410, X-ray data from XMM-Newton. 

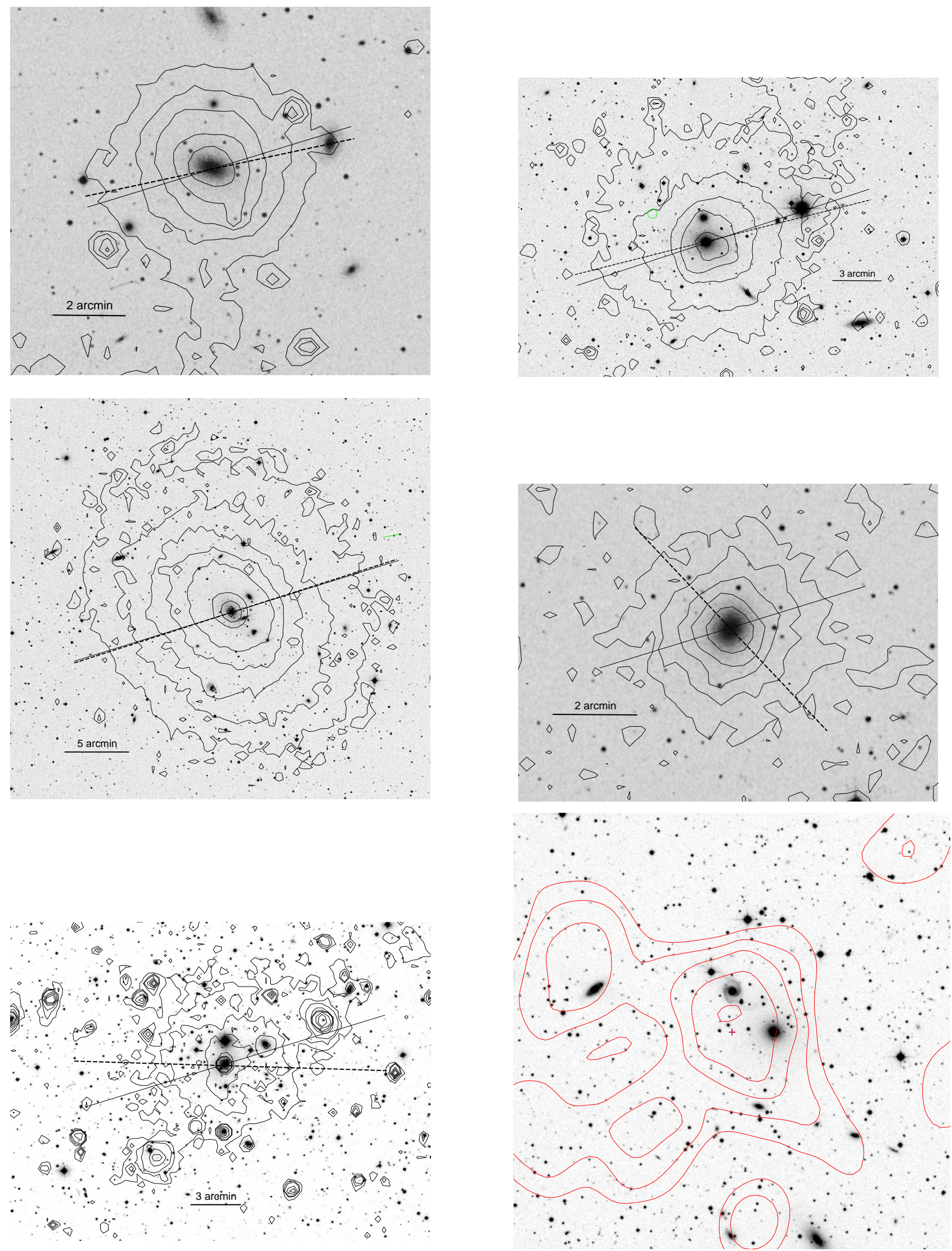

Fig. A.2. Images of Perseus-Pisces SC members continued. Upper left: RXCJ0123.1+3327, NGC 499, X-ray data from XMM-Newton; upper right: RXCJ0123.6+3315, NGC 507, X-ray data from XMM-Newton; middle left: RXCJ0152.7+3609, A 262, X-ray data from XMM-Newton; middle right: RXCJ0200.2+3126, NGC 777, X-ray data from XMM-Newton; lower left: R0222.7+4301, UGC 1841, X-ray data from XMM-Newton; lower right: RXCJ0238.7+4138, NGC 996, X-ray data from RASS. 

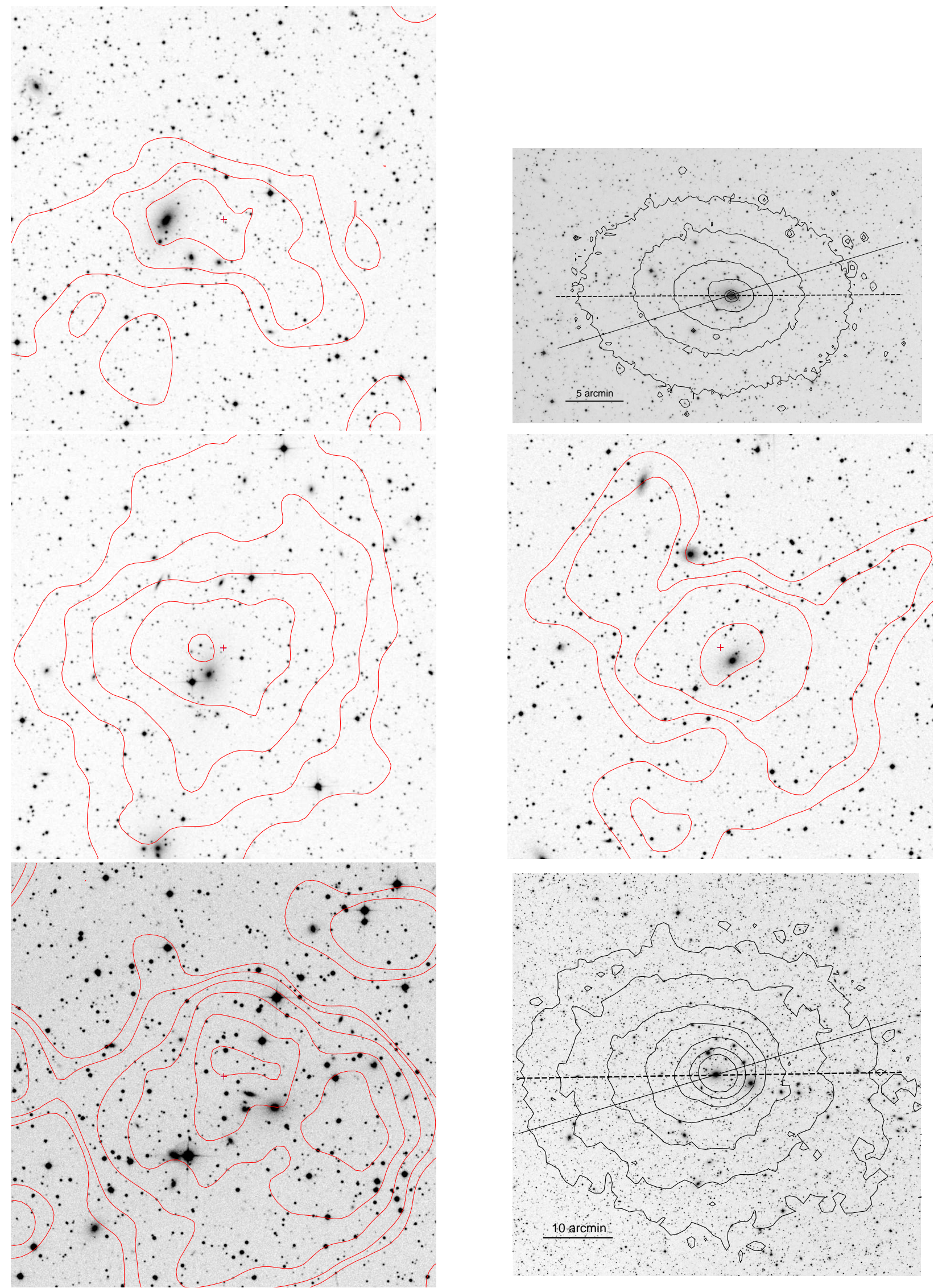

Fig. A.3. Images of Perseus-Pisces SC members continued. Upper left: RXCJ0249.5+4658, IC 257, X-ray data from RASS; upper right: RXCJ0254.4+4134, NGC 1129, AWM7, X-ray data from XMM-Newton; middle left: RXCJ0300.7+4428, CIZAJ0300.7+4427, X-ray data from RASS; middle right: RXCJ0309.9+4207, UGC 2562, X-ray data from RASS; lower left: RXCJ0310.3+4250, X-ray data from RASS; lower right: RXCJ0319.7+4130, A 426, Perseus, X-ray data from ROSAT pointed observation. 

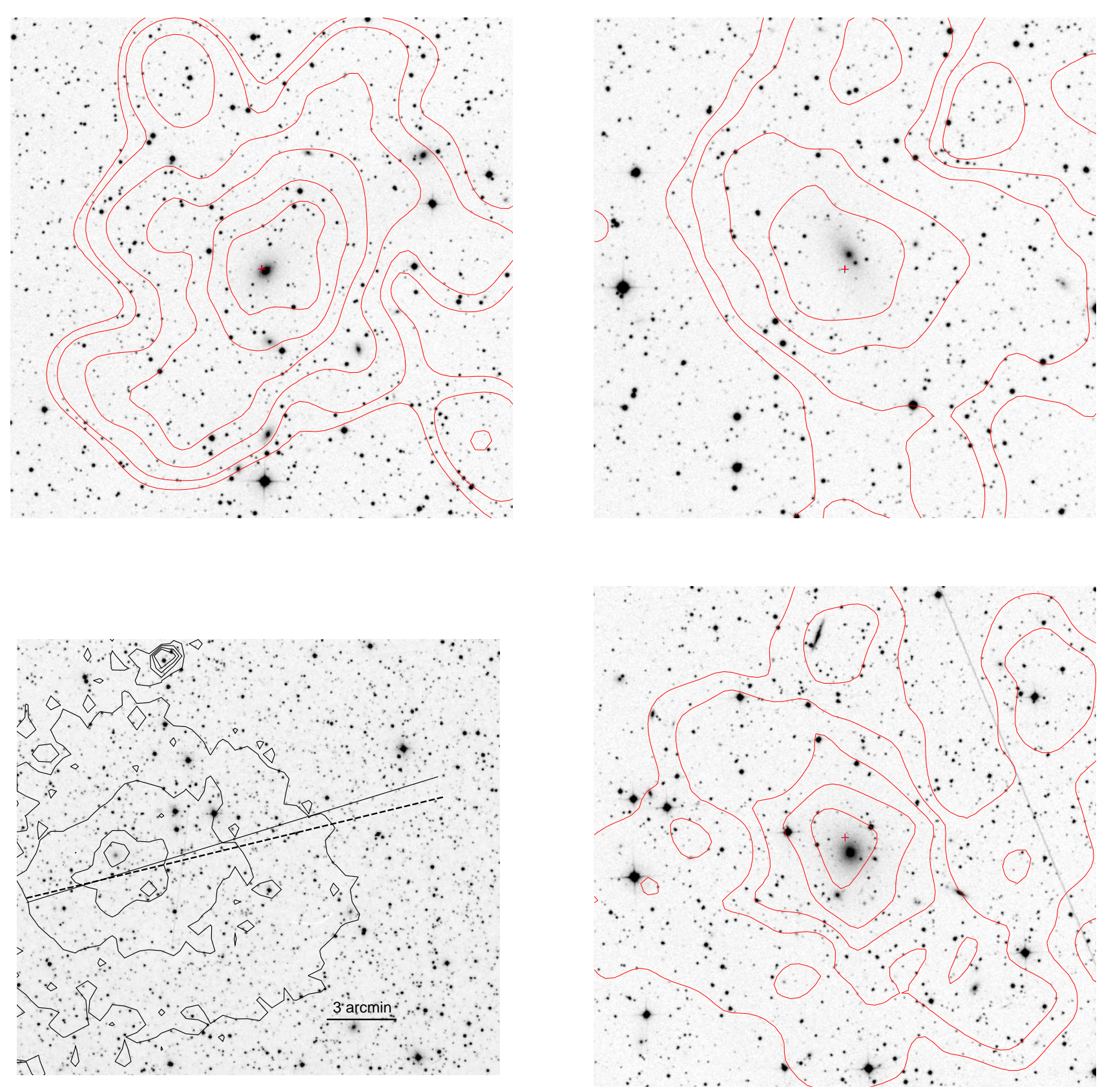

Fig. A.4. Images of Perseus-Pisces SC members continued. Upper left: RXCJ0348.1+4212, MCG +07-08-033, X-ray data from RASS; upper right: RXCJ0421.8+3607, UGC 03021, X-ray data from RASS; lower left: RXCJ0450.0+4501, 3C 129, X-ray data from XMM-Newton; lower right: RXCJ0547.2+5052, UGC 03355, X-ray data from RASS. 
H. Böhringer et al.: Perseus-Pisces supercluster

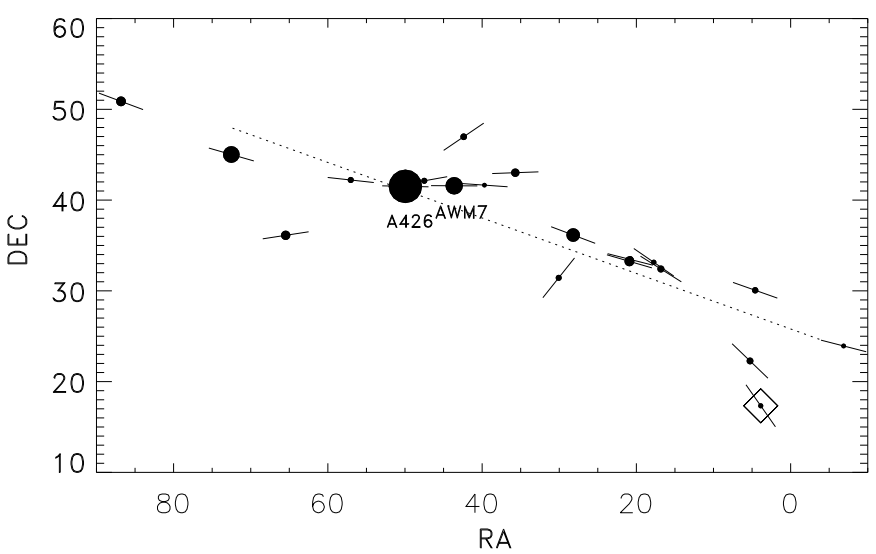

Fig. A.5. Distribution of the members of the Perseus SC in equatorial coordinates with main axis of the SC (dashed line) and the direction of the tidal forces acting on each cluster (short solid lines).

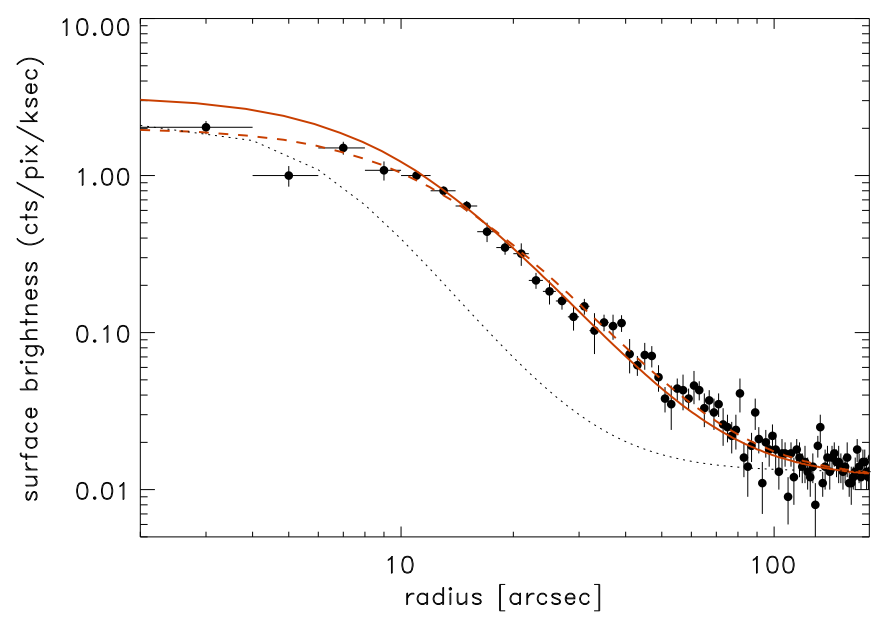

Fig. A.6. X-ray surface brightness profile of NGC 410 traced by the MOS detectors of XMM-Newton (data points with error bars). Also shown is the profile of the point spread function (dotted curve). The red lines show the best fit of a beta-model, where the dashed line gives the source profile convolved with the point spread function, which was fitted, and the solid line the deconvolved profile. 
A\&A 651, A16 (2021)
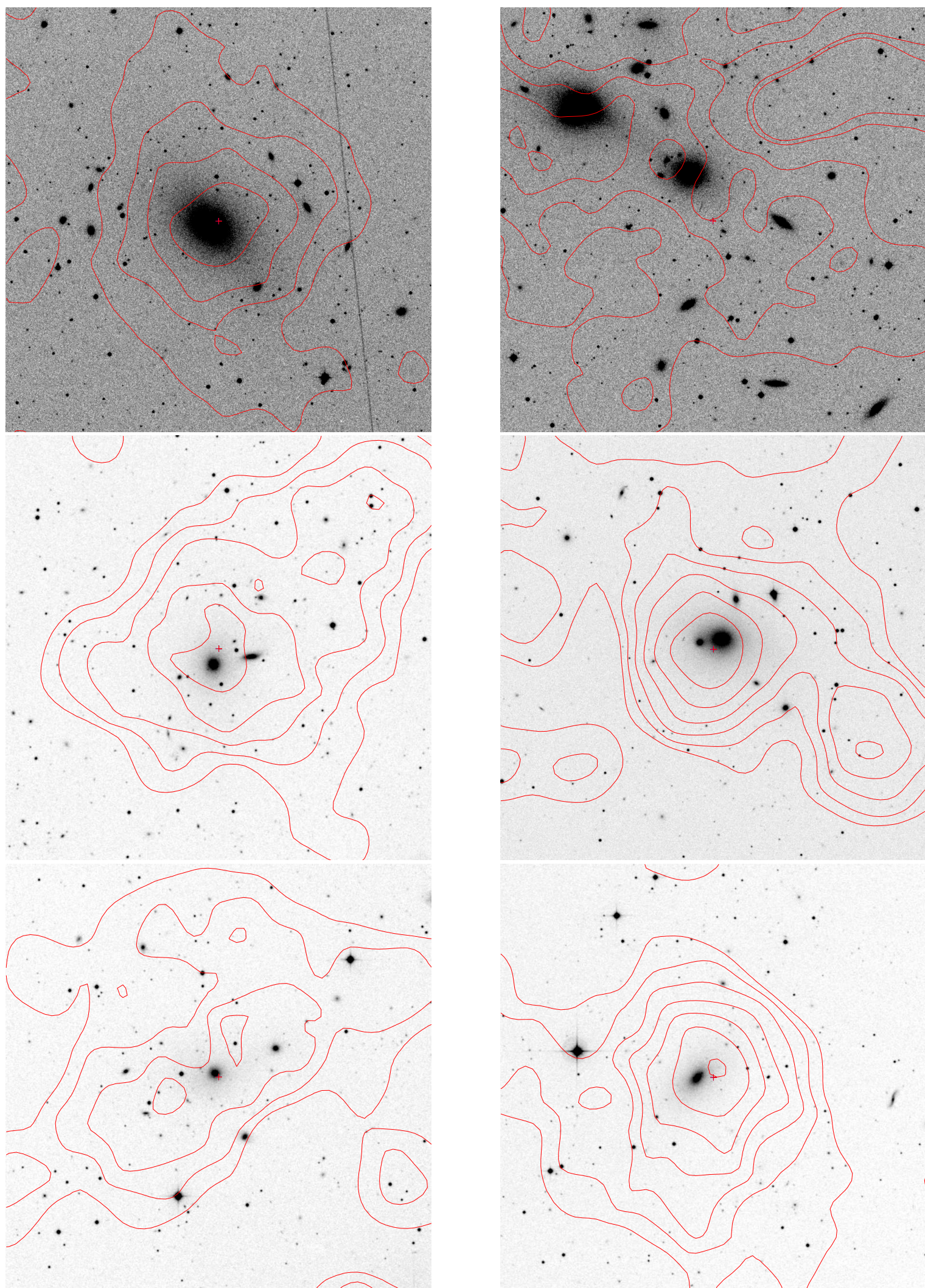

Fig. A.7. X-ray contours overlayed on optical images from the DSS database for the members of the Southern Great Wall. All X-ray data are from RASS. Upper left: RXCJ0125.5+0145, NGC 533; upper right: RXCJ0125.6-0124, A 194; middle left: RXCJ0149.2+1303, NGC 677; middle right: RXCJ0156.3+0537, NGC 741; lower left: RXCJ0231.9+0114, UGC 2005; lower right: RXCJ0252.8-0116, NGC 1132. 
H. Böhringer et al.: Perseus-Pisces supercluster

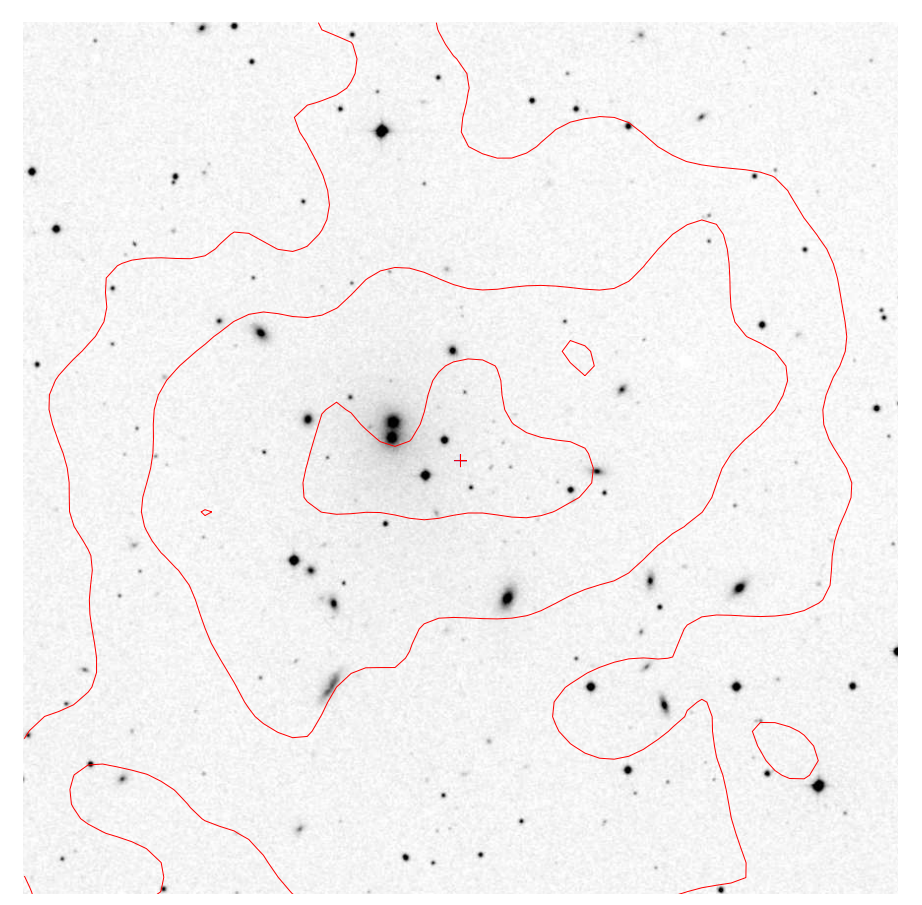

Fig. A.8. Continuation of images of the Southern Great Wall. SXCJ0257.6+0600, A 400, with data from RASS. 